\title{
Toward an improved definition of the genetic and tumor spectrum associated with $S D H$ germ-line mutations
}

\author{
Lucie Evenepoel, MS'1-3, Thomas G. Papathomas, MD², Niels Krol, BS², Esther Korpershoek, PhD², \\ Ronald R. de Krijger, MD, PhD ${ }^{2,4}$, Alexandre Persu, MD' and Winand N.M. Dinjens, PhD²
}

The tricarboxylic acid, or Krebs, cycle is central to the cellular metabolism of sugars, lipids, and amino acids; it fuels the mitochondrial respiratory chain for energy generation. In the past decade, mutations in the Krebs-cycle enzymes succinate dehydrogenase, fumarate hydratase, and isocitrate dehydrogenase have been documented to be causally involved in carcinogenesis. This review is focused on the relationship between $S D H$ mutations and the carcinogenic phenotype. The succinate dehydrogenase complex catalyzes the oxidation of succinate to fumarate; mutations in its subunits $S D H A, S D H B, S D H C$, and $S D H D$, and in the assembly factor $S D H A F 2$, result in syndromes with distinct tumor types, including pheochromocytoma/paraganglioma, gastrointestinal stromal tumor, and, less often, renal-cell carcinoma and pituitary adenoma. In this study we collected all previously reported $S D H$ mutations with the aim of defining their nature and tumor spectrum. In addition, genotype-phenotype correlations as well as mechanisms of biallelic inactivation were analyzed in the $S D H$-deficient setting. Finally, we performed bioinformatics analysis using SIFT, Polyphen2, and Mutation Assessor to predict the functional impact of nonsynonymous mutations. The prediction of the latter was further compared with available SDHA and/ or SDHB immunohistochemistry data.

Genet Med advance online publication 13 November 2014

Key Words: genetics; immunohistochemistry; paragangliomas; pheochromocytomas; succinate dehydrogenase
The tricarboxylic acid (TCA) cycle enzyme succinate dehydrogenase $(\mathrm{SDH})$ is a heterotetramer protein complex consisting of four subunits encoded by nuclear genes. These include SDHA and SDHB, which form the catalytic domain, and SDHC and SDHD, which anchor the complex to the inner mitochondrial membrane. ${ }^{1}$ The assembly factors, SDHAF1 and SDHAF2, ensure both structural and functional integrity of the complex. ${ }^{2,3}$ $\mathrm{SDH}$, also called mitochondrial complex II, is the only enzyme involved in both the electron transport chain and the TCA cycle, where it catalyzes the oxidation of succinate to fumarate. ${ }^{1}$ The TCA cycle is central to the metabolism of sugars, lipids, and amino acids and is a major source of adenosine triphosphate in cells. In addition, the cycle also seems to be involved in tumorigenesis; enzymes of the TCA cycle are involved in the pathogenesis of several tumor types. $S D H$ mutations have been involved in the etiopathogeny of pheochromocytomas (PCCs), paragangliomas (PGLs), gastrointestinal stromal tumors (GISTs), renal-cell carcinomas (RCCs), and pituitary adenomas (PAs). ${ }^{1,24-9}$ In addition, mutations in fumarate hydratase $(F H)$, another member of the TCA cycle and which catalyzes the hydration of fumarate to malate, predispose to tumor formation, including RCCs, cutaneous and uterine leiomyomas, and PCCs/PGLs. ${ }^{10,11}$ Finally, isocitrate dehydrogenase (IDH), which catalyzes the oxidative decarboxylation of isocitrate, is frequently mutated in specific types of cartilaginous tumors, hematological malignancies, and gliomas. ${ }^{12-14}$ The currently known mechanisms underlying tumorigenesis linked to defects in the TCA cycle are well reviewed. ${ }^{15,16}$ Defects in the $S D H, F H$, and $I D H$ genes inhibit prolyl hydroxylases, leading to decreased hydroxylation of hypoxia-inducible factor- $\alpha$. This results in activation of the hypoxia pathway, which supports tumor formation by activating angiogenesis, glucose metabolism, cell motility, and cell survival. Furthermore, defects in these enzymes lead to epigenetic alterations through an accumulation of oncometabolites inhibiting $\alpha$-ketoglutarate-dependent dioxygenases, which are involved in DNA and histone demethylation. In addition to $S D H$-associated tumorigenesis, constitutional complex II deficiencies caused by $S D H A, S D H B, S D H D$, and SDHAF1 mutations may also lead to Leigh syndrome, infantile leukodystrophies, and cardiomyopathy.,17-19

In the current review, our aim is to report all currently known $S D H$ mutations and define their nature and spectrum in $\mathrm{SDH}$ related tumors, including PCCs/PGLs, GISTs, RCCs, and PAs, as well as in other unusual tumors arising in $S D H$ mutation carriers. We performed bioinformatics analysis using SIFT, Polyphen2, and Mutation Assessor and compared the results with those of SDHA/SDHB immunohistochemistry (IHC) to predict the functional impact of nonsynonymous mutations. Finally, we explored and report here the nature of the second hit in all tumors arising in the SDH deficiency setting. 


\section{MATERIALS AND METHODS}

The search for $S D H$ mutations was performed using the Leiden Open Variation Database (http://www.lovd.nl/3.0/ home) and publications indexed in PubMed (http://www. ncbi.nlm.nih.gov/pubmed) from 2000, the year of the first published mutation in a paraganglioma, to April 2014. ${ }^{1,2,4-6}$ The following search terms were used: SDHA, SDHB, SDHC, $S D H D$, and $S D H A F 2$, in combination with the tumor type names pheochromocytomas, paragangliomas (sympathetic and parasympathetic), gastrointestinal stromal tumors, renal-cell carcinomas, and pituitary adenomas. ${ }^{20}$

With the aim of predicting the functional impact of the $S D H$ mutations on the corresponding proteins, a bioinformatics analysis was performed using the ANNOVAR software. ${ }^{21}$ First, the variants found in the literature were checked in the Leiden Open Variation Database, and the genomic positions were obtained using Mutalyzer. ${ }^{20,22}$ Then the variants were annotated using ANNOVAR with the following databases: hg19, RefSeq with HGVS as the nomenclature type, 1000 Genomes 2012APR, NonFlagged dbSNP138, CLINVAR20140211, and COSMIC68. ${ }^{23-25} 1000$ Genomes 2012APR and NonFlagged dbSNP138, describing only single-nucleotide polymorphisms with a frequency lower than $1 \%$, were used to determine whether the changes were reported as single-nucleotide polymorphisms. ${ }^{25,26}$ CLINVAR20142011 describes the associations between DNA variations and human health, whereas COSMIC68 encompasses somatic mutations in cancers. ${ }^{27,28}$ Finally, the scores of SIFT, Polyphen-2, and Mutation Assessor were calculated by means of the ANNOVAR software to assess the potential functionally damaging impact of the amino acid changes. ${ }^{29-31}$ The Consensus Deleteriousness software, which combines information derived from SIFT, Polyphen-2, and Mutation Assessor, was used to predict the functionality of nonsynonymous mutations. ${ }^{32}$ In addition, we calculated the Combined Annotation Dependent Depletion score (http:// cadd.gs.washington.edu/score), which is another predictor for the pathogenicity of an amino acid change and includes the previous prediction models. ${ }^{33}$

\section{RESULTS}

\section{Pheochromocytomas and paragangliomas}

In total, 445 different germ-line mutations in the $S D H$ genes were reported in the literature. Of these, 403 mutations have been described in PCCs/PGLs: 211 (52\%) in SDHB, 141 (35\%) in SDHD, $42(10 \%)$ in SDHC, 7 (2\%) in SDHA, and $2(1 \%)$ in SDHAF2 (Figure 1). Missense mutations occurred most frequently in all genes, with the exception of $S D H D$, in which more frameshift mutations were observed (34\%) (Figure 1). In total, 141 missense mutations (35\%) and 104 frameshift mutations (26\%) were observed in PCCs/PGLs (Figure 1). One somatic missense mutation has been identified in $S D H B$ (c.299C $>\mathrm{T}$, p.S100F). Seventy-eight mutations (19\%) were described in malignant tumors: $59(76 \%)$ in $S D H B, 15(19 \%)$ in $S D H D$, and $4(5 \%)$ in $S D H C$ (Figure 2). In these genes, no hotspot mutations were related to malignant behavior.

\section{Gastrointestinal stromal tumors, renal-cell carcinomas, and} pituitary adenomas

Fifty-five germ-line mutations were identified in the GISTs, of which 26 (47\%) occurred in SDHA, 14 (25\%) in SDHB, 9 (16\%) in $S D H C$, and $6(11 \%)$ in SDHD (Figure 1). Missense mutations occurred most frequently in each gene except SDHD, in which frameshift mutations were the most common (34\%; Figure 1). Thirty mutations were found in RCCs: 25 (83\%) in $S D H B, 3(10 \%)$ in SDHC, and $2(7 \%)$ in SDHD (Figure 1). Most mutations were missense mutations (47\%). In addition, eight SDH mutations were reported in PAs: three (38\%) occurred in SDHD, two each $(25 \%)$ in SDHB and SDHC, and one $(12 \%)$ in SDHA (Figure 1). Five of the PA-related mutations (62\%) were missense. Three somatic mutations were observed in SDHA: one in a GIST (c.113A>T, p.D38V) and two in one PA (c.725_736del and c.989_990insTA).

\section{Mutations occurring in two or more tumor types}

Fifty-three germ-line mutations have been described in more than one tumor type: 29 (55\%) occurred in SDHB, 10 (19\%) in $S D H C, 10$ (19\%) in SDHD, and 4 (7\%) in SDHA (Figure 3a). Twenty-three $(44 \%)$ were missense mutations. In total, 19 mutations (36\%) were observed in PCCs/PGLs and RCCs, 16 (30\%) caused PCCs/PGLs and GISTs, and 6 (11\%) were found in PCCs/PGLs and PAs. In addition, five mutations (9\%) were reported in PCCs/PGLs, GISTs, and RCCs. Furthermore, five mutations (9\%) were identified in at least one causally related tumor type (PCC/PGL, GIST, RCC, and PA) and one unusual tumor type (thyroid carcinoma, uterine myoma, breast cancer, and neuroblastomas), and two mutations (4\%) have been identified in the four causally related tumors (Figure 3a). Seventeen different mutations-of which seven were missense-that caused multiple causally related tumors (PCCs/PGLs, GISTs, RCCs, and PAs) in single patients were identified: seven (41\%) occurred in $S D H B$, seven (41\%) in $S D H D$, and three $(18 \%)$ in SDHC (Figure 3b).

\section{Mutations occurring exclusively in GISTs, RCCs, or PAs}

Of 445 different germ-line mutations reported in the SDH genes, 39 (9\%) were exclusively found in GISTs, RCCs, or PAs and not in PCCs/PGLs: 24 (61\%) occurred in SDHA, 8 (21\%) in $S D H B, 5$ (13\%) in SDHC, and $2(5 \%)$ in SDHD (Figure 4a). In addition, 33 mutations (85\%) were observed in GISTs, 5 (13\%) in RCCs, and 1 (2\%) in PAs (Figure 4a). In total, $44 \%$ of the mutations reported exclusively in GISTs, RCCs, and PAs were missense (Figure 4a).

\section{Mutations associated with tumors occurring rarely in the context of SDH deficiency}

Twenty-three different germ-line SDH mutations were identified in patients with other tumor types, including thyroid carcinomas, neuroblastomas, and breast cancer, in addition to their PCC/PGL. Of these 23 mutations, 9 (39\%) occurred in SDHB, $8(35 \%)$ in SDHD, $4(17 \%)$ in SDHC, and $2(9 \%)$ in SDHA (Figure 4b). In total, 35\% were missense mutations (Figure $4 b$ ). 
a

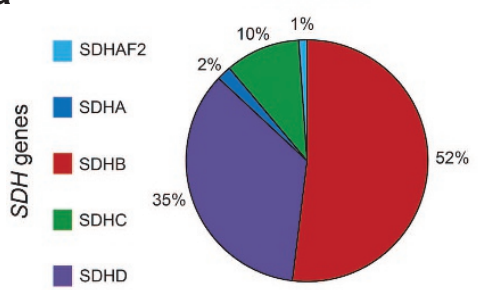

GIST

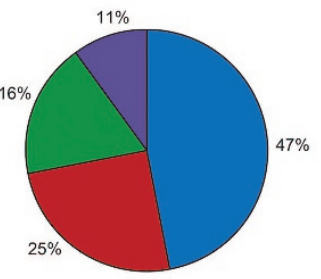

RCC
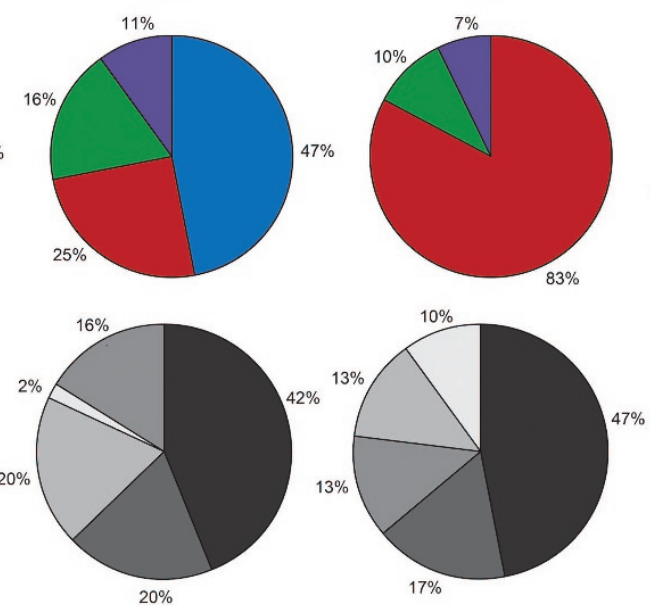
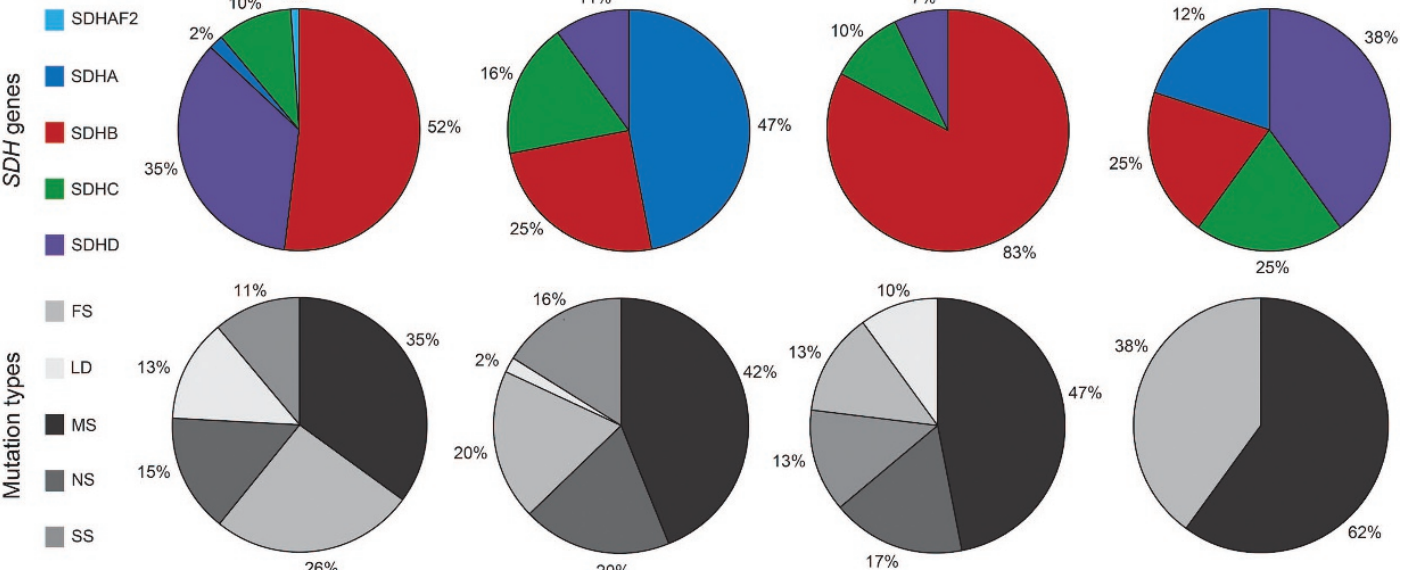

b
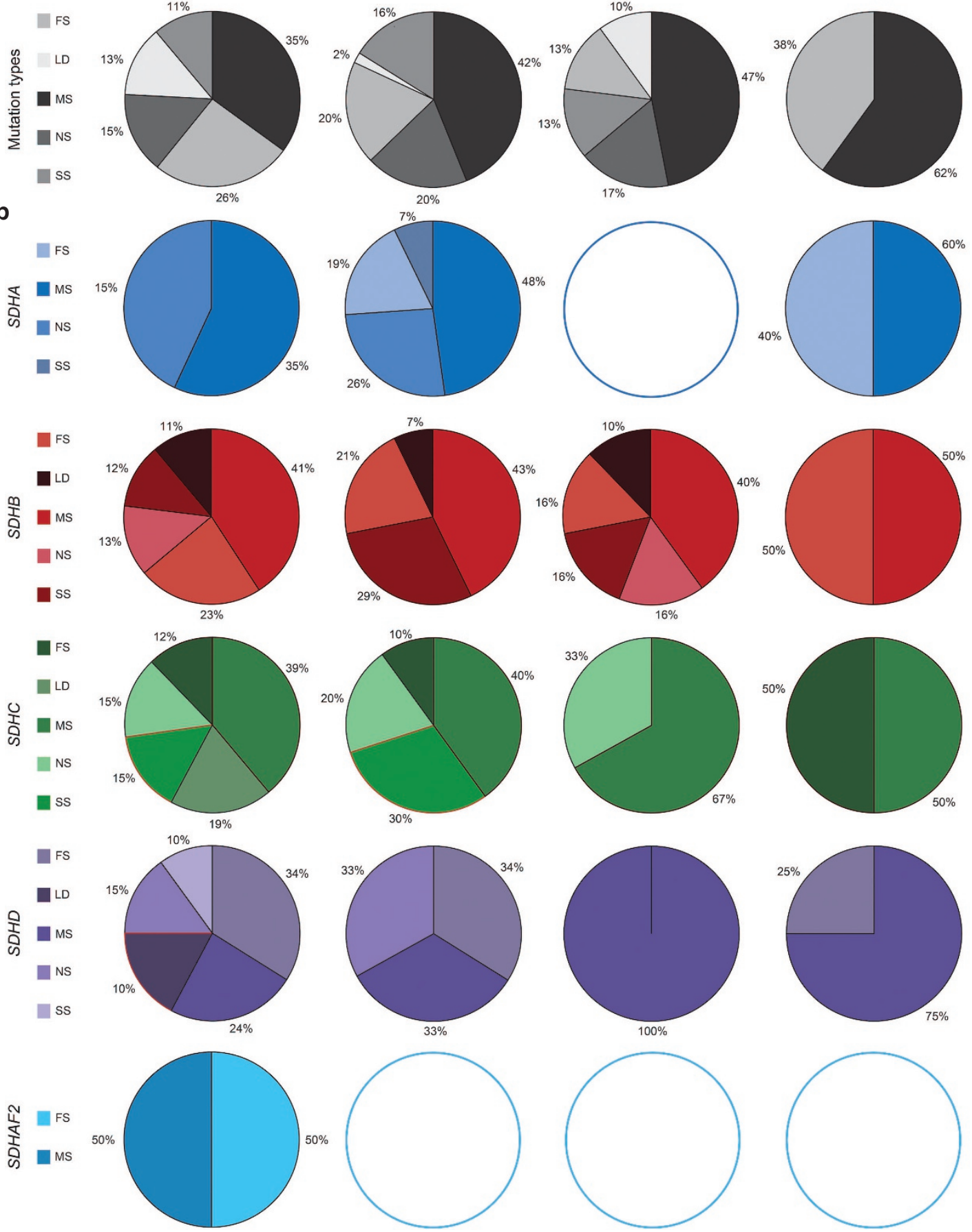

Figure 1 Spectrum and distribution of SDH germ-line mutations as reported in SDH-deficient tumors including pheochromocytomas (PCCs)/ paragangliomas (PGLs), gastrointestinal stromal tumors (GISTs), renal-cell carcinomas (RCCs), and pituitary adenomas (PAs). (a) Percentages of mutations in each SDH gene and of the different types of mutations: frameshift deletion/duplication/insertion (FS), large deletion (LD), missense (MS), nonsense (NS), and splice site (SS). (b) Percentages of the different mutation types for each SDH gene. 
a
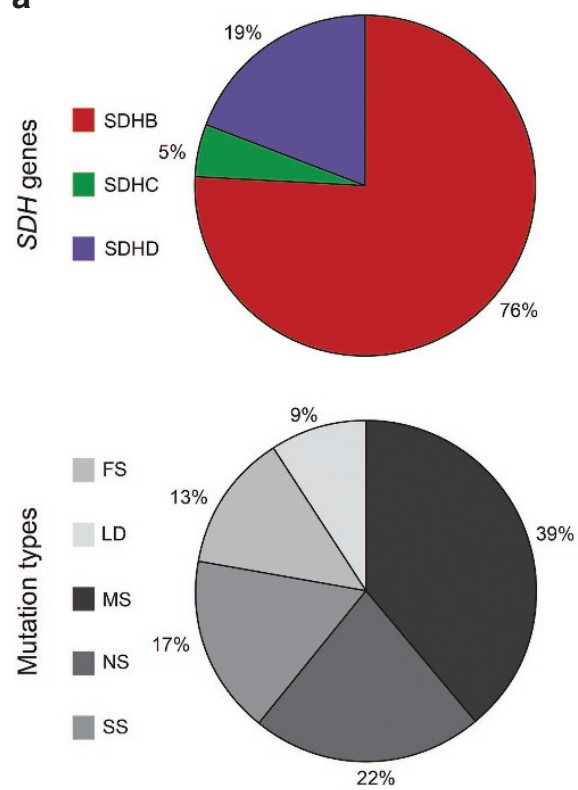

b
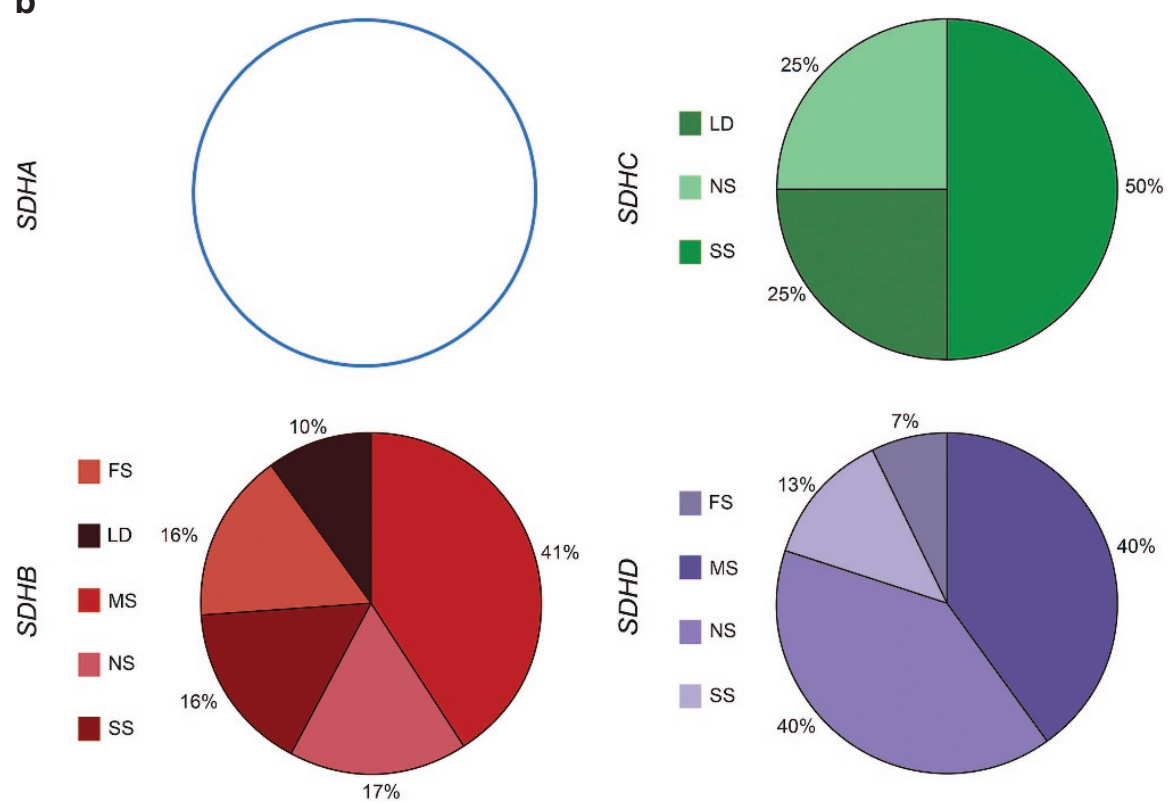

Figure 2 Spectrum and distribution of SDH germ-line mutations as reported in malignant pheochromocytoma (PCC)/paragangliomas (PGL). (a) Percentages of mutations in each SDH gene and of the different types of mutations reported in malignant PCCs/PGLs. (b) Percentages of different mutation types identified in malignant tumors for each SDH gene.

Establishing the causal link between these mutations and these tumors was not possible because IHC data were not reported. However, one study reported a patient suffering from a PGL and a testicular seminoma who had an $S D H D$ mutation $(\mathrm{c} .129 \mathrm{G}>\mathrm{A}$, p.W43X) and showed loss of the wild-type (WT) allele in the tumor (Supplementary Table S2 online).

In silico analysis of the functional impact of $S D H$ mutations An in silico analysis was performed to predict the functional impact of each SDH nonsynonymous mutation. Three scores were assessed: SIFT, Polyphen2, and Mutation Assessor. In addition, the mutations were associated with the immunohistochemical results of the SDHA and SDHB staining because this technique has been proven to reliably predict the presence of an $S D H$ mutation: A negative $S D H B$ staining is predictive of the presence of a mutation in any $S D H$ subunit, with a sensitivity of $100 \%$ and a specificity of $84 \%$, whereas a negative SDHA staining predicts an SDHA mutation with a sensitivity of $100 \%$ and a specificity of $97 \% .^{34,35}$ The IHC results were available for 145 previously reported mutations. In the vast majority (134, or $92 \%$ ), the IHC pattern was consistent with the prediction obtained by bioinformatics (Supplementary Table S1 online). A total of 129 mutations were predicted to be damaging to the protein and showed consistent negative SDHA and/or SDHB staining (Supplementary Table S1 online). The remaining five mutations were predicted to be nondeleterious and showed positive SDHA and/or SDHB staining (Supplementary Table S1 online). Nevertheless, some exceptions were reported. Two SDHA mutations reported in GISTs (c.113A $>$ T, p.Asp38Val and c.1969G $>$ A, p.Val657Ile) were predicted to be nondamaging but showed negative SDHA and SDHB staining (Table 1).
Moreover, nine mutations predicted to be deleterious had positive SDHA and/or SDHB IHC results, although they were expected to have negative results (Table 1). For the mutations showing nonconsistent IHC results, we calculated the Combined Annotation Dependent Depletion. ${ }^{33}$ A Cscore of 10 indicates that the variant is part of the $10 \%$ most damaging substitutions in the human genome. Considering as damaging a variant with a Cscore $\geq 15$ is therefore suggested (http://cadd. gs.washington.edu/info). The Consensus Deleteriousness and the Combined Annotation Dependent Depletion scores reach the same prediction for each mutation.

\section{Second hit}

The nature of the second hit has been reported for 85 SDH mutations (Supplementary Table S2 online). Inactivation of the WT allele by loss of heterozygosity (LOH) has been observed 62 times (73\%) and by somatic mutations in 12 cases (14\%). In addition, eight mutations (9\%) co-occurred with retention of heterozygosity, and epigenetic inactivation of the second allele was reported once. A combination of multiple second hits has been found for two SDHA mutations: c.91C $>\mathrm{T}$, p.Arg $31^{\star}$ and c.1873C > T, p.His625Tyr (Supplementary Table S2 online).

\section{DISCUSSION}

In this review, we present an overview of all currently reported $S D H$ mutations and analyze potential genotype-phenotype correlations with regard to the development of neoplasia. In addition, we performed bioinformatics analysis on the functional impact of the reported $S D H$ mutations and compared the results with available SDHA and SDHB IHC results. Furthermore, we explored the nature of the second hit observed 
a Mutations reported in $>1 \mathrm{SDH}$ deficient tumor types

A. 1

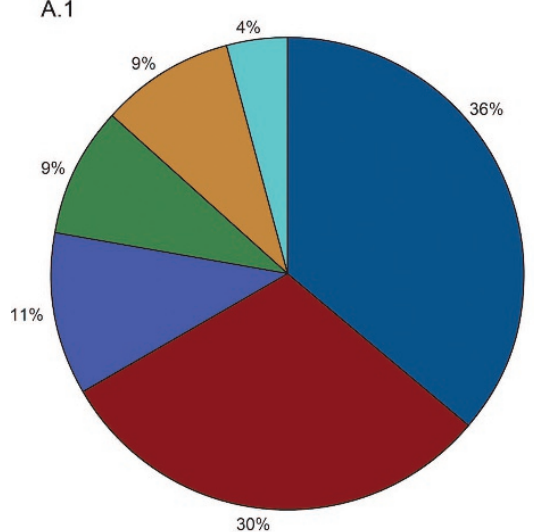

$\mathrm{PCC} / \mathrm{PGL}+\mathrm{RCC}$

PCC/PGL + GIST

$P C C / P G L+P A$

$\mathrm{PCC} / \mathrm{PGL}+\mathrm{GIST}+\mathrm{RCC}$

Unusual tumor types

PCC/PGL + GIST+ RCC + PA

A. 3
A. 2
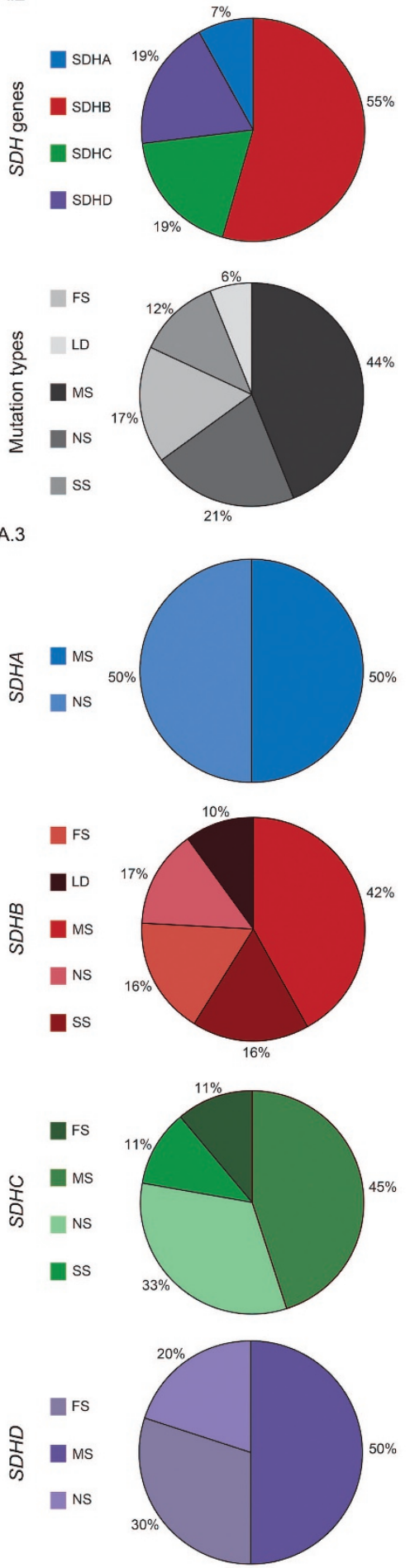

b Mutations reported in $>1 S D H$ deficient tumors in single patients

B.1
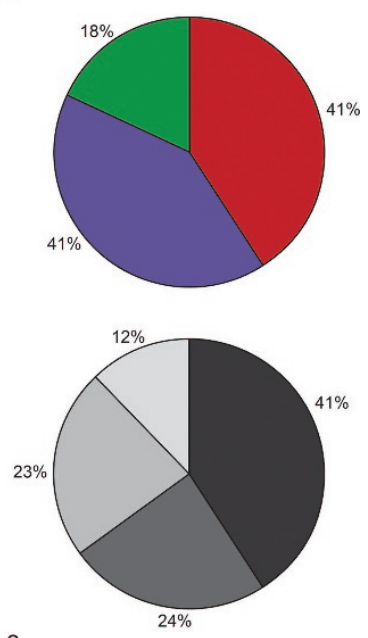

B.2
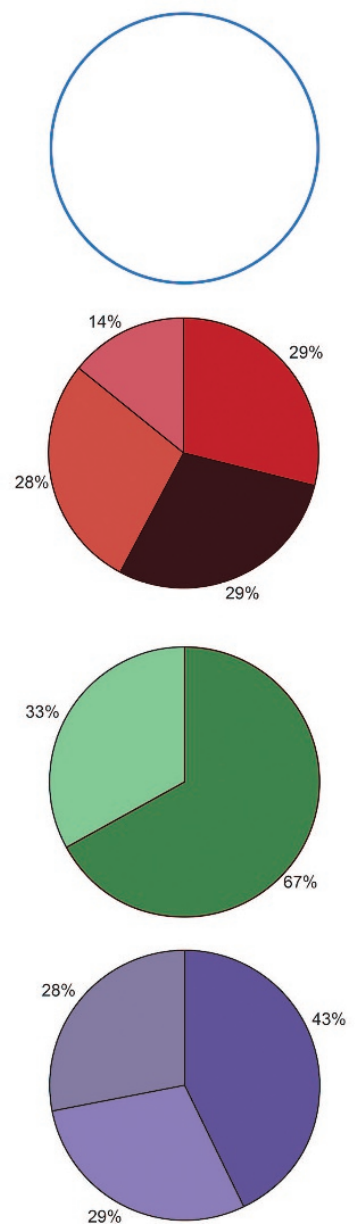

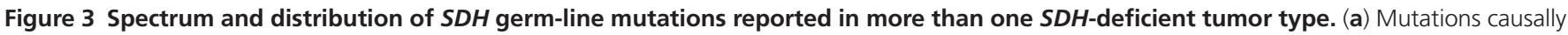

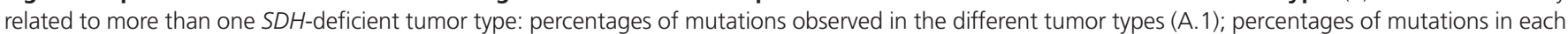

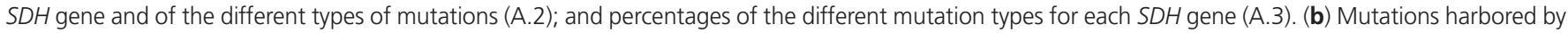

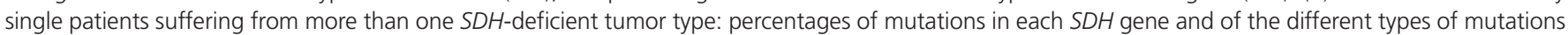
(B. 1) and percentages of the different mutation types for each SDH gene (B.2). 
a Spectrum and distribution of SDH mutations exclusively identified in GISTs, RCCs, and PAs

A. 1

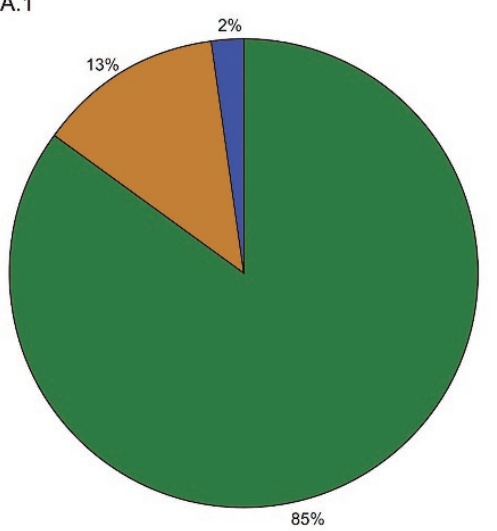

GIST

RCC

PA
A.2

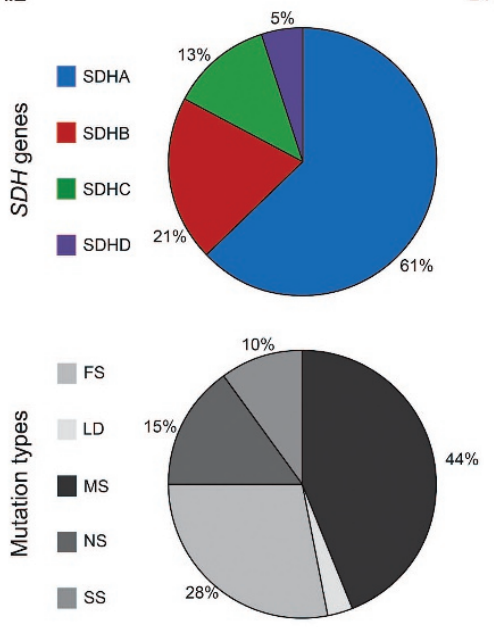

A.3

B.1

क प MS

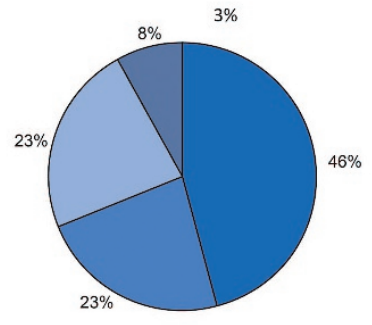

b

Spectrum and distribution of $S D H$

mutations as reported in unusual tumors
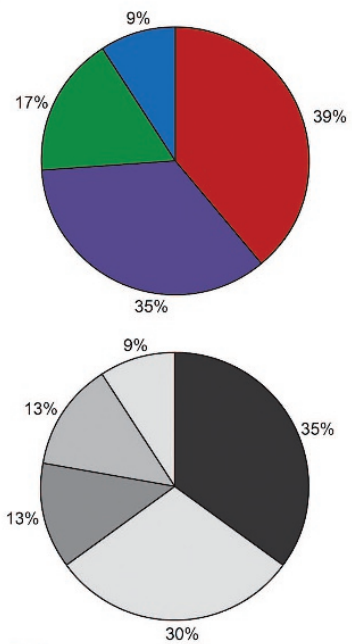

B.2
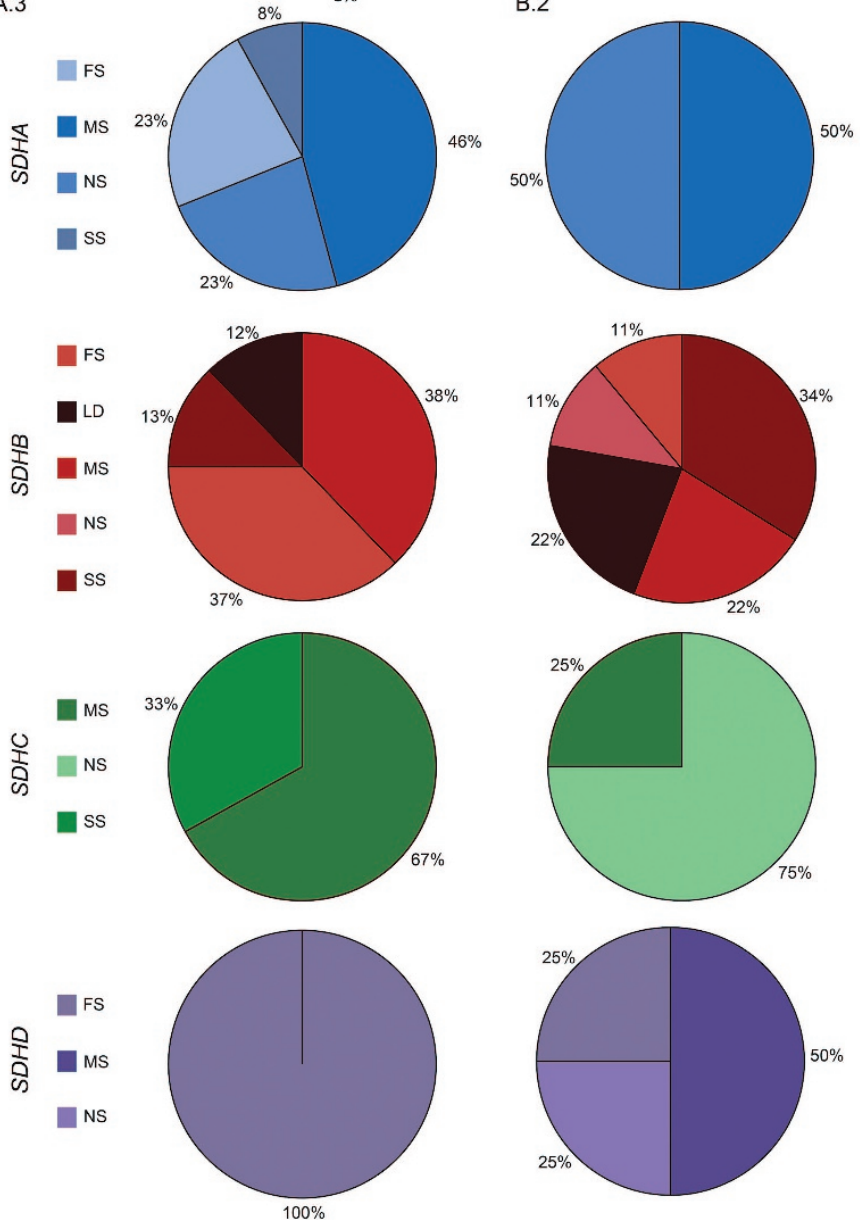

Figure 4 Spectrum and distribution of SDH germ-line mutations exclusively identified in gastrointestinal stromal tumors (GISTs), renal-cell carcinomas (RCCs), or pituitary adenomas (PAs) and as reported in tumors occurring rarely in the context of SDH deficiency. (a) Spectrum and distribution of SDH mutations exclusively identified in GISTs, RCCS, and PAs: percentages of mutations as reported only in GISTs, RCCS, and PAs in each of those tumor types (A.1); percentages of the mutations reported in the related tumor types except pheochromocytomas (PCCs)/paragangliomas (PGLs) in each SDH gene and of the different types of mutations (A.2); and percentages of the different mutation types identified in the related tumor types except PCCs/PGLs for each SDH gene (A.3). (b) Spectrum and distribution of SDH mutations as reported in tumors occurring rarely in the context of SDH deficiency: percentages of mutations in each SDH gene and of the different types of mutations reported in different tumors including thyroid carcinomas and neuroblastomas (B.1), and percentages of different mutation types identified in these different tumors for each SDH gene (B.2). 
in tumors arising in both germ-line and somatic $S D H$ mutation carriers.

\section{Genotype-phenotype correlation analysis}

Two genotype-phenotype correlations were highlighted. The first was the high frequency of $S D H B$ mutations in malignant PCCs/PGLs: $76 \%$ of the mutations identified in malignant cases occurred in $S D H B$ (Figure 1a). The association between $S D H B$ mutations and malignancy has been reported many times, but only a few studies provide mechanistic insights. ${ }^{36-40}$ Taking the results of those studies together, the association between malignancy and $S D H B$ mutations might be due to a hypermethylator phenotype that was observed in SDHB-mutated PCCs/PGLs in particular. ${ }^{37}$ This hypermethylation would lead to a high epigenetic silencing of genes that play a role in differentiation and in the epithelial-to-mesenchymal transition. ${ }^{36,37}$ By contrast, SDHA mutations seem to show no association with malignancy. This could be because of the small numbers of SDHA-mutated cases known to date; only seven different $S D H A$ mutations have been identified in PCCs/PGLs, and there is a lack of follow-up of patients with apparently benign disease (Figure 1a).

The rarity of SDHA-associated PCC/PGL might be related to the low frequency of $5 \mathrm{p} 15$ loss seen in PCC and PGL, which is the chromosomal location of SDHA. By contrast, PCC and PGL show high frequencies of loss of $1 \mathrm{p} 36$ and $11 \mathrm{q} 23$, the chromosomal areas where $S D H B$ and $S D H D$ are located, respectively. ${ }^{6}$

The second strong genotype-phenotype correlation concerns the association of GIST with SDHA; $47 \%$ of the mutations reported in this tumor type occurred in $S D H A$ (Figure 1a). It was recently shown that SDHA mutations occur in $~ 30 \%$ of $S D H$ deficient GISTs. These GISTs occur in patients at an older age and have a lower female-to-male ratio than other $S D H$-deficient malignancies. No differences in histology between SDHA WT and mutant GISTs have been discerned. ${ }^{41}$ Most $S D H$-deficient GISTs encompass tumors arising in Carney-Stratakis syndrome and the Carney triad, in pediatric GISTs, and in a subset of apparently sporadic KIT/PDGFRA WT GISTs. ${ }^{41,42}$ These GISTs are characterized by unique clinicopathological features and biological properties: (i) female preponderance; (ii) gastric location (predilection for the distal stomach/antrum); (iii) common multifocality; (iv) a multinodular/plexiform growth pattern; (v) epithelioid cytomorphology, either pure or combined with a spindle-cell component; (vi) SDHA and/or SDHB immunonegativity; (vii) KIT (and DOG1) immunopositivity despite the lack of KIT/PDGFRA mutations; (viii) metastatic potential (often to lymph nodes); (ix) a relatively indolent clinical course, even in the presence of metastatic disease; and (x) insensitivity to imatinib. ${ }^{41,42}$

With regard to RCCs and PAs, the number of reported mutations is too low (30 and 10, respectively) to draw reliable conclusions about any potential genotype-phenotype correlations, although it must be noted that $83 \%$ of the mutations identified in RCCs occurred in $S D H B$ (Figure 1a). SDH-deficient RCC is a unique subtype of RCC that has been recently accepted as a provisional entity in the 2013 International Society of Urological
Pathology Vancouver Classification..$^{43} \mathrm{SDH}$-deficient RCCs display a slight male predisposition, present as bilateral tumors in $25 \%$ of cases, and have stereotypical morphologic features, that is, solid or focally cystic growth, uniform cytology with eosinophilic flocculent cytoplasm, intracytoplasmic vacuolations and inclusions, round to oval low-grade nuclei, and SDHB immunonegativity. ${ }^{44,45}$ SDHA-negative IHC was reported once, but no mutational screening of $S D H A$ was performed..$^{44}$ Despite the fact that $S D H$-deficient RCC is capable of progression, metastatic disease is rare in the absence of high-grade nuclear atypia or coagulative necrosis. ${ }^{44,45}$

Finally, $S D H$-deficient PAs seem likely to be prolactin-secreting (and/or prolactin-immunopositive) and, to a lesser extent, growth hormone-secreting macroadenomas. They are usually accompanied by a personal history of PCC/PGL and have a slight male predisposition. ${ }^{46,47}$

\section{In silico analysis association with SDHA/SDHB IHC}

The prediction of the impact of an $S D H$ mutation was associated with available SDHA and/or SDHB IHC data, as SDHA/ SDHB IHC is predictive of the presence of an $S D H$ mutation. Indeed, a negative staining for SDHB on IHC indicates a mutation in any of the $S D H$ subunits, whereas a negative SDHA staining on IHC exclusively predicts the presence of an SDHA mutation. In the majority of cases, bioinformatics analysis, predicting the effect of nonsynonymous mutations, was consistent with the available IHC results (Supplementary Table S1 online). However, some exceptions were reported. Two previously reported SDHA mutations (c.113A>T; p.Asp38Val and c.1969G $>A$; p.Val657Ile) were predicted as nondeleterious and had a Cscore lower than 15 but were negative for SDHA and $\mathrm{SDHB}^{48,49}$ (Table 1). The p.Val657Ile mutation was reported in COSMIC, and both mutations have been reported in a sample population: p.Asp38Val; rs34635677; minor allele frequency: $\mathrm{T}$ $=0.012 / 26$ and p.Val657Ile; rs6962; minor allele frequency: $\mathrm{A}=$ 0.161/351 (dbSNP; http://www.ncbi.nlm.nih.gov/SNP/). These discrepancies could reflect the fact that these variants might affect the epitope recognized by the SDHA antibody, explaining the absence of staining on IHC. ${ }^{48,49}$ However, this is highly unlikely because both SDHA and SDHB are negative on IHC, indicating a deleterious effect of the variant on the protein. ${ }^{48,49}$ Finally, these discrepancies could also be due to staining artifacts. Nonconsistent IHC results were also observed for 9 mutations predicted as damaging and having a Cscore higher than 15 but having positive IHC results (Table 1). Three discrepancies occurred in SDHA and showed SDHB-negative and SDHApositive IHC (c.562C > T; p.Arg188Trp, c.818C > T; p.Thr273Ile, and c.1361C $>$ A; p.Ala454Glu). ${ }^{41,50}$ Two other SDHA mutations (c.778G >A; p.Gly260Arg and c.1873C > T; p.His625Tyr) were positive for SDHB and SDHA on IHC. ${ }^{51,52}$ Another one was in $S D H C$ (c.380A $>$ G; p.His127Arg), and the last three were in $S D H D$ (c.14G>A; p.Trp5 ${ }^{\star}$, c.149A $>$ G; p.His50Arg, and c.274G>T; p.Asp92Tyr) ${ }^{46,53-55}$ Among these SDHD mutations, one resulted in a stop codon (p.Trp5 $5^{\star}$ ), which cannot be managed by the prediction tools we used but can be considered to 


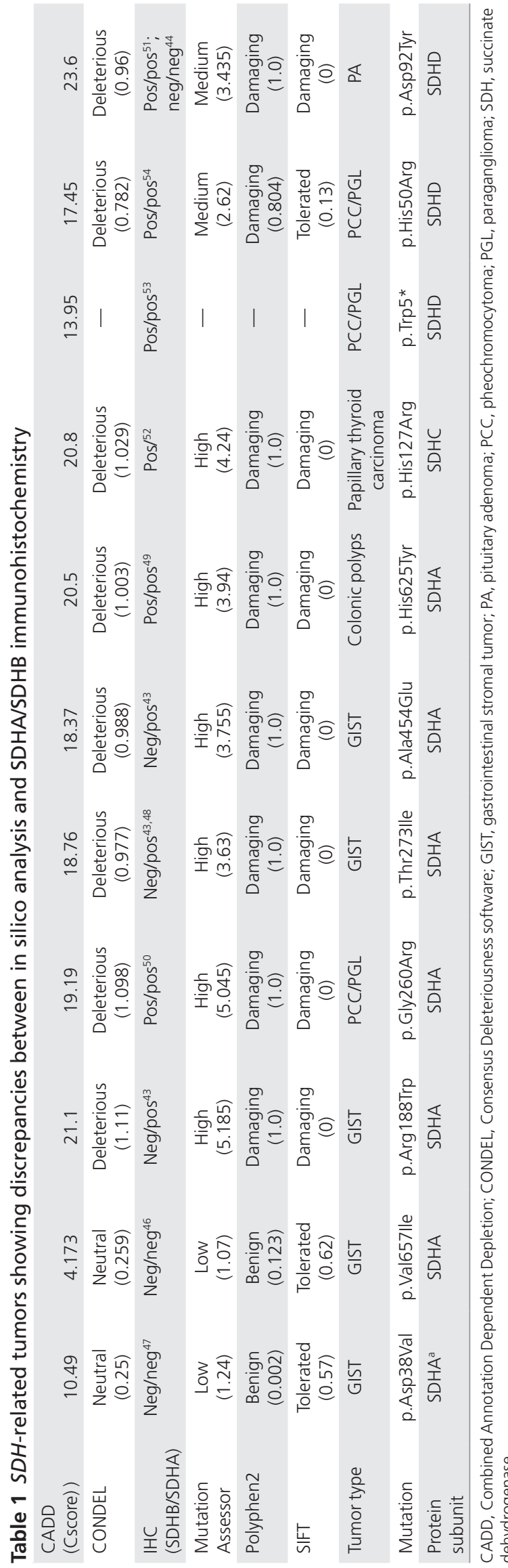

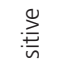

:

be damaging because the mutation results in a severely truncated protein. This nonsense variant was identified in a patient suffering from Carney-Stratakis syndrome, with the PGL showing positive SDHB on IHC and the GIST presenting with a negative SDHB on IHC, potentially indicating two different second hit events in the SDHD gene. ${ }^{54}$ Another SDHD mutation, p.Asp92Tyr, was reported in two patients affected by PAs; one PA showed an SDHB-negative IHC and the other PA still expressed SDHB. ${ }^{46}$ Nevertheless, this variant was observed in many PCCs/PGLs that had corresponding SDHB-negative IHC. ${ }^{34}$ Therefore, these few discrepant results might be due to staining artifacts, a wrong interpretation, an undetected mutation, or another mechanism of inactivation.

Although SDHA and SDHB IHC reliably identifies the presence of $S D H$ mutations, the previous findings show that positive staining has been observed in tumors with mutations predicted as damaging. Therefore, in addition to IHC, bioinformatics analysis should be used as a tool to support the definition of the pathogenicity of $S D H$ variants of unknown significance. Functional studies could provide more proof to identify the pathogenicity of uncertain variants.

\section{Mechanisms of biallelic inactivation}

$S D H$ genes function as tumor suppressor genes (TSGs) with $\mathrm{LOH}$ as the predominant mechanism of inactivation (and somatic mutation less frequently) of the WT allele (Supplementary Table S2 online). However, different mechanisms, including biallelic inactivating $S D H$ mutations and epigenetic silencing, can coexist (e.g., SDH-deficient GISTs) ${ }_{48,49,56-60}$ (Supplementary Table S2 online). Notably, two sisters with an SDHA mutation (c.91C $>$ T, p.Arg31*) were affected by SDHA/SDHB-immunonegative GISTs; one displayed LOH, whereas the other showed retention of heterozygosity, probably indicating a different mechanism of inactivation, such as promoter methylation of the WT allele. ${ }^{60}$ However, an undetected mutation seems to be the most likely mechanism. In addition, haploinsufficient and/or dominant negative effects for the $S D H$ genes could be another mechanism of inactivation and have been indicated in particular cases: (i) bilateral adrenal medullary hyperplasia associated with a germ-line $S D H B$ mutation (c.587G $>$ A, p.Cys196Tyr) showing retention of heterozygosity $^{61}$; (ii and iii) SDHA/SDHB immunonegative GIST and PA in patients with $S D H A$ mutations (c.91C>T, p.Arg31*; c.1873C $>$ T, p.His625Tyr) displaying either retention of heterozygosity or paradoxical loss of the mutated SDHA allele, respectively ${ }^{51,60}$; (iv) PCCs without loss of the WT SDHD allele arising in patients with SDHA mutations (c.341A $>$ G, p.Tyr114Cys; c.441delG, p.Gly148Alafs $\left.{ }^{\star} 20\right)^{62}$; and (v) somatic SDHD inactivation associated with consistent reduction of transcript levels in neural crest-derived, neuroendocrine, and gastrointestinal tumors. $^{38,63} \mathrm{~A}$ recent report showed that somatic SDHC hypermethylation might be the cause of the Carney triad, a multitumoral syndrome of unknown etiology affecting five organs: paraganglia (PGL), adrenal gland (adrenocortical adenoma and PCC), lung (chondroma), stomach (GIST), and esophagus 
(leiomyoma). ${ }^{64,65}$ This suggests that epigenetic silencing of $S D H$ could also be an important mechanism involved in tumor development and could explain SDH deficiency in the absence of germ-line mutations.

Tumorigenesis caused by haploinsufficiency seems unlikely because $S D H D, S D H D / H 19$, and $S D H B$ knockout mice, as well as conditional or inducible tissue-specific $S D H D$ knockouts, do not develop tumors or any other genotype-related pathology except for a slight carotid body hyperplasia ${ }^{66-69}$ (Judith Favier, personal communication).

\section{Tumor suppressor effect in $\mathbf{S D H}$-related tumorigenesis}

TSG-related, tissue-specific tumorigenesis may be attributed to a complex interplay, but novel evidence points to a model of TSG loss-driven tumorigenesis, whereas haploinsufficiency might act synergistically with oncogenic signals in a tissuespecific manner. ${ }^{70-72}$ With regard to $S D H$-related oncogenesis, it has been suggested that the tumor phenotypes could be affected by the size of $S D H$ deletions because they can encompass closely located TSGs and compromise their function, which could lead to unusual phenotypes. ${ }^{73,74}$ In addition, some research suggests that germ-line and somatic interactions could account for the clinical variability observed among carriers of an identical $S D H$ germ-line mutation, including those within the same family. ${ }^{46,75-77}$ In this context, the occurrence of $S D H$-related tumors is extremely rare in the absence of a germ-line mutation. Indeed, only a few cases have been reported: one extra-adrenal PGL harboring a somatic $S D H B$ mutation (c.299C $>$ T, p.Ser100Phe) and a somatic $S D H D$ mutation in a PCC (c.242C >T, p.Pro81Leu), both displaying $\mathrm{LOH}$; one PA harboring two somatic SDHA mutations (c.725_736del; c.989_990insTA); and tumors from four patients with the Carney triad showing a DNA hypermethylation of $S D H C$. $^{47,65,78,79}$

Although familial PCC/PGL syndromes were thought to predispose only to PCCs and PGLs, different tumors such as GISTs, RCCs, and PAs have expanded the tumor spectrum associated with $S D H$ mutations. ${ }^{77}$ No patient has developed all tumor types, indicating an incomplete penetrance of the syndrome similar to that seen in the Carney triad..$^{64}$ To explain the issue of cell specificity, Hoekstra and Bayley ${ }^{69}$ pointed toward differences of cell types either in their requirements for adenosine triphosphate, redox agents, and TCA cycle-generated metabolic intermediates during development and/or throughout life or in their response to cancerous transformation. Importantly, both SDH mutant PCCs/PGLs as well as GISTs have been recently shown to display a hypermethylator phenotype, exposing a vital interplay among succinate metabolism, epigenetic disruptions, and tissue-specific tumorigenesis. ${ }^{37,65,80}$

\section{Conclusion}

Familial PCC/PGL syndromes were initially thought to predispose only to PCCs and PGLs, but several tumors, including GISTs, RCCs, and PAs, have expanded the $S D H$-associated tumor spectrum. Extensive clinical variability can be expected, even among carriers of an identical $S D H$ germ-line mutation, with tumor phenotypes being only partially expressed, as in the Carney-Stratakis dyad.

Previous reports of genotype-phenotype correlations showed that $S D H B$ mutations are associated with a high risk of malignancy, and SDHD mutations are associated with headand-neck PGL. ${ }^{81,82}$ Our results show an additional strong genotype-phenotype correlation, as SDHA mutations seem to be associated with $S D H$-deficient GISTs.

In addition, the absence of SDHA and/or SDHB expression in tumor tissue on IHC is an indicator of the functional absence of one of the SDH genes. Therefore, SDHA/SDHB IHC, along with in silico analysis and tumor $\mathrm{LOH}$ analysis, seem to be valuable tools in determining the potential pathogenicity of $S D H$ variants and therefore have significant implications in the new era of comprehensive next-generation sequencing-based approaches for the analysis of PCC/PGL susceptibility genes. Although questions about some incidental cases remain, based on data from the current comprehensive literature, we demonstrated that the $S D H$ genes behave as bona fide TSGs with biallelic inactivation through combinations of germ-line mutations, somatic mutations, $\mathrm{LOH}$, and epigenetic silencing, contributing to a specific spectrum of malignancies.

\section{SUPPLEMENTARY MATERIAL}

Supplementary material is linked to the online version of the paper at http://www.nature.com/gim

\section{ACKNOWLEDGMENTS}

The authors acknowledge the Seventh Framework Programme (FP7/2007-2013), under grant agreement 259735 (ENS@T-Cancer), and the Fonds de la Recherche Scientifique-FNRS for supporting this project.

\section{DISCLOSURE}

The authors declare no conflict of interest.

\section{REFERENCES}

1. Baysal BE, Ferrell RE, Willett-Brozick JE, et al. Mutations in SDHD, a mitochondrial complex II gene, in hereditary paraganglioma. Science 2000;287:848-851.

2. Hao HX, Khalimonchuk O, Schraders M, et al. SDH5, a gene required for flavination of succinate dehydrogenase, is mutated in paraganglioma. Science 2009;325:1139-1142.

3. Ghezzi D, Goffrini P, Uziel G, et al. SDHAF1, encoding a LYR complex-II specific assembly factor, is mutated in SDH-defective infantile leukoencephalopathy. Nat Genet 2009;41:654-656.

4. Astuti D, Latif F, Dallol A, et al. Gene mutations in the succinate dehydrogenase subunit SDHB cause susceptibility to familial pheochromocytoma and to familial paraganglioma. Am J Hum Genet 2001;69:49-54.

5. Niemann S, Müller U. Mutations in SDHC cause autosomal dominant paraganglioma, type 3. Nat Genet 2000;26:268-270.

6. Burnichon N, Brière JJ, Libé R, et al. SDHA is a tumor suppressor gene causing paraganglioma. Hum Mol Genet 2010;19:3011-3020.

7. Vanharanta S, Buchta M, McWhinney SR, et al. Early-onset renal cell carcinoma as a novel extraparaganglial component of SDHB-associated heritable paraganglioma. Am J Hum Genet 2004;74:153-159.

8. Xekouki P, Stratakis CA. Succinate dehydrogenase (SDHx) mutations in pituitary tumors: could this be a new role for mitochondrial complex II and/or Krebs cycle defects? Endocr Relat Cancer 2012;19:C33-C40.

9. Pasini B, MCWhinney SR, Bei T, et al. Clinical and molecular genetics of patients with the Carney-Stratakis syndrome and germline mutations of the genes 
coding for the succinate dehydrogenase subunits SDHB, SDHC, and SDHD. Eur J Hum Genet 2008;16:79-88.

10. Tomlinson IP, Alam NA, Rowan AJ, et al.; Multiple Leiomyoma Consortium. Germline mutations in $\mathrm{FH}$ predispose to dominantly inherited uterine fibroids, skin leiomyomata and papillary renal cell cancer. Nat Genet 2002;30:406-410.

11. Castro-Vega $L$, Buffet $A$, De Cubas $A A$, et al. Germline mutations in FH confer predisposition to malignant pheochromocytomas and paragangliomas. Hum Mol Genet 2014;23:2440-2446.

12. Yan $\mathrm{H}$, Parsons DW, Jin G, et al. IDH1 and IDH2 mutations in gliomas. N Eng/ J Med 2009;360:765-773.

13. Schaap FG, French PJ, Bovée JV. Mutations in the isocitrate dehydrogenase genes IDH1 and IDH2 in tumors. Adv Anat Pathol 2013;20:32-38.

14. Zou Y, Zeng Y, Zhang DF, Zou SH, Cheng YF, Yao YG. IDH1 and IDH2 mutations are frequent in Chinese patients with acute myeloid leukemia but rare in other types of hematological disorders. Biochem Biophys Res Commun 2010;402:378-383.

15. Raimundo N, Baysal BE, Shadel GS. Revisiting the TCA cycle: signaling to tumor formation. Trends Mol Med 2011;17:641-649.

16. Desideri E, Vegliante R, Ciriolo MR. Mitochondrial dysfunctions in cancer: genetic defects and oncogenic signaling impinging on TCA cycle activity. Cancer Lett 2014; doi: 10.1016/j.canlet.2014.02.023 e-pub ahead of print 12 March 2014.

17. Alston CL, Davison JE, Meloni F, et al. Recessive germline SDHA and SDHB mutations causing leukodystrophy and isolated mitochondrial complex II deficiency. J Med Genet 2012;49:569-577.

18. Levitas A, Muhammad E, Harel G, et al. Familial neonatal isolated cardiomyopathy caused by a mutation in the flavoprotein subunit of succinate dehydrogenase. Eur J Hum Genet 2010;18:1160-1165.

19. Bourgeron $T$, Rustin $P$, Chretien $D$, et al. Mutation of a nuclear succinate dehydrogenase gene results in mitochondrial respiratory chain deficiency. Nat Genet 1995:11:144-149.

20. Fokkema IF, Taschner PE, Schaafsma GC, Celli J, Laros JF, den Dunnen JT. LOVD v.2.0: the next generation in gene variant databases. Hum Mutat 2011;32:557-563.

21. Wang K, Li MY, Hakonarson H. ANNOVAR: functional annotation of genetic variants from high-throughput sequencing data. Nucleic Acids Res 2010;38:e164

22. Wildeman M, van Ophuizen E, den Dunnen JT, Taschner PE. Improving sequence variant descriptions in mutation databases and literature using the Mutalyzer sequence variation nomenclature checker. Hum Mutat 2008;29:6-13. Accessed 1 April, 2014.

23. National Center for Biotechnology Information. Chapter 18, The Reference Sequence (RefSeq) Project. http://www.ncbi.nlm.nih.gov/books/NBK21091. National Library of Medicine: Bethesda, MD, 2002.

24. den Dunnen JT, Antonarakis SE. Mutation nomenclature extensions and suggestions to describe complex mutations: a discussion. Hum Mutat 2000;15:7-12.

25. Altshuler DM, Durbin RM, Abecasis GR, et al. An integrated map of genetic variation from 1,092 human genomes. Nature 2012;491:56-65.

26. National Center for Biotechnology Information. Database of Single Nucleotide Polymorphisms (dbSNP). National Library of Medicine: Bethesda, MD.

27. Landrum MJ, Lee JM, Riley GR, et al. ClinVar: public archive of relationships among sequence variation and human phenotype. Nucleic Acids Res 2014;42(Database issue):D980-D985.

28. Forbes SA, Bindal N, Bamford S, et al. COSMIC: mining complete cancer genomes in the Catalogue of Somatic Mutations in Cancer. Nucleic Acids Res 2011;39(Database issue):D945-D950.

29. Ng PC, Henikoff S. Predicting deleterious amino acid substitutions. Genome Res 2001;11:863-874.

30. Adzhubei IA, Schmidt S, Peshkin L, et al. A method and server for predicting damaging missense mutations. Nat Methods 2010;7:248-249.

31. Reva B, Antipin Y, Sander C. Determinants of protein function revealed by combinatorial entropy optimization. Genome Biol 2007;8:R232.

32. González-Pérez A, López-Bigas N. Improving the assessment of the outcome of nonsynonymous SNVs with a consensus deleteriousness score, Condel. Am J Hum Genet 2011;88:440-449.

33. Kircher M, Witten DM, Jain P, O'Roak BJ, Cooper GM, Shendure J. A general framework for estimating the relative pathogenicity of human genetic variants. Nat Genet 2014;46:310-315.

34. van Nederveen FH, Gaal J, Favier J, et al. An immunohistochemical procedure to detect patients with paraganglioma and phaeochromocytoma with germline SDHB, SDHC, or SDHD gene mutations: a retrospective and prospective analysis. Lancet Oncol 2009;10:764-771.
35. Korpershoek E, Favier J, Gaal J, et al. SDHA immunohistochemistry detects germline SDHA gene mutations in apparently sporadic paragangliomas and pheochromocytomas. J Clin Endocrinol Metab 2011;96:E1472-E1476.

36. Loriot C, Burnichon N, Gadessaud N, et al. Epithelial to mesenchymal transition is activated in metastatic pheochromocytomas and paragangliomas caused by SDHB gene mutations. J Clin Endocrinol Metab 2012;97:E954-E962.

37. Letouzé $E$, Martinelli $C$, Loriot $C$, et al. SDH mutations establish a hypermethylator phenotype in paraganglioma. Cancer Cell 2013;23:739-752.

38. Pasini B, Stratakis CA. SDH mutations in tumorigenesis and inherited endocrine tumours: lesson from the phaeochromocytoma-paraganglioma syndromes. J Intern Med 2009;266:19-42.

39. Gimenez-Roqueplo AP, Favier J, Rustin P, et al.; COMETE Network. Mutations in the SDHB gene are associated with extra-adrenal and/or malignant phaeochromocytomas. Cancer Res 2003;63:5615-5621.

40. King KS, Prodanov T, Kantorovich V, et al. Metastatic pheochromocytoma/ paraganglioma related to primary tumor development in childhood or adolescence: significant link to SDHB mutations. J Clin Oncol 2011;29:41374142.

41. Miettinen M, Killian JK, Wang ZF, et al. Immunohistochemical loss of succinate dehydrogenase subunit A (SDHA) in gastrointestinal stromal tumors (GISTs) signals SDHA germline mutation. Am J Surg Pathol 2013;37:234-240.

42. Doyle LA, Hornick JL. Gastrointestinal stromal tumours: from KIT to succinate dehydrogenase. Histopathology 2014;64:53-67.

43. Srigley JR, Delahunt B, Eble JN, et al.; ISUP Renal Tumor Panel. The International Society of Urological Pathology (ISUP) Vancouver Classification of renal neoplasia. Am J Surg Patho/ 2013;37:1469-1489.

44. Williamson SR, Eble JN, Amin MB, et al. Succinate dehydrogenase-deficient renal cell carcinoma: detailed characterization of 11 tumors defining a unique subtype of renal cell carcinoma. Mod Patho/ 2014; e-pub ahead of print 18 July 2014.doi: $10.1038 /$ modpathol.2014.86

45. Gill AJ, Hes O, Papathomas T, et al. Succinate dehydrogenase (SDH)-deficient renal carcinoma: a morphologically distinct entity: a clinicopathologic series of 36 tumors from 27 patients. Am J Surg Pathol 2014; e-pub ahead of print 14 July 2014.

46. Papathomas TG, Gaal J, Corssmit EP, et al. Non-pheochromocytoma (PCC)/ paraganglioma (PGL) tumors in patients with succinate dehydrogenaserelated PCC-PGL syndromes: a clinicopathological and molecular analysis. Eur J Endocrinol 2014;170:1-12.

47. Gill AJ, Toon CW, Clarkson A, et al. Succinate dehydrogenase deficiency is rare in pituitary adenomas. Am J Surg Patho/ 2014;38:560-566.

48. Dwight T, Benn DE, Clarkson A, et al. Loss of SDHA expression identifies SDHA mutations in succinate dehydrogenase-deficient gastrointestinal stromal tumors. Am J Surg Pathol 2013;37:226-233.

49. Italiano A, Chen CL, Sung YS, et al. SDHA loss of function mutations in a subset of young adult wild-type gastrointestinal stromal tumors. BMC Cancer 2012;12:408.

50. Belinsky MG, Rink L, von Mehren M. Succinate dehydrogenase deficiency in pediatric and adult gastrointestinal stromal tumors. Front Onco/ 2013;3:117.

51. Dwight T, Mann K, Benn DE, et al. Familial SDHA mutation associated with pituitary adenoma and pheochromocytoma/paraganglioma. J Clin Endocrinol Metab 2013;98:E1103-E1108.

52. Mensenkamp AR, van Gassen K, Kusters B, Kunst DH, Bongers MHF, Lenders JWM. Prevalence of SDHA mutations and associated clinical spectrum among patients with pheochromocytomas, extra adrenal paragangliomas and glomus tumors. Paper presented at ENSAT Meeting, Budapest, Hungary, 22-23 November 2013.

53. Malinoc A, Sullivan M, Wiech T, et al. Biallelic inactivation of the SDHC gene in renal carcinoma associated with paraganglioma syndrome type 3. Endocr Relat Cancer 2012;19:283-290.

54. Tenorio Jiménez C, Izatt L, Chang F, Moonim MT, Carroll PV, McGowan BM. Carney Stratakis syndrome in a patient with SDHD mutation. Endocr Pathol 2012;23:181-186

55. Sjursen W, Halvorsen $\mathrm{H}$, Hofsli E, et al. Mutation screening in a Norwegian cohort with pheochromocytoma. Fam Cancer 2013;12:529-535.

56. Pantaleo MA, Astolfi A, Indio V, et al. SDHA loss-of-function mutations in KIT-PDGFRA wild-type gastrointestinal stromal tumors identified by massively parallel sequencing. J Natl Cancer Inst 2011;103:983-987.

57. Wagner AJ, Remillard SP, Zhang YX, Doyle LA, George S, Hornick JL. Loss of expression of SDHA predicts SDHA mutations in gastrointestinal stromal tumors. Mod Pathol 2013;26:289-294.

58. Belinsky MG, Rink L, Flieder DB, et al. Overexpression of insulin-like growth factor 1 receptor and frequent mutational inactivation of SDHA in wild-type 
SDHB-negative gastrointestinal stromal tumors. Genes Chromosomes Cancer 2013;52:214-224.

59. Celestino R, Lima J, Faustino A, et al. A novel germline SDHB mutation in a gastrointestinal stromal tumor patient without bona fide features of the Carney-Stratakis dyad. Fam Cancer 2012;11:189-194.

60. Oudijk L, Gaal J, Korpershoek E, et al. SDHA mutations in adult and pediatric wild-type gastrointestinal stromal tumors. Mod Pathol 2013;26:456-463.

61. Grogan RH, Pacak K, Pasche L, Huynh TT, Greco RS. Bilateral adrenal medullary hyperplasia associated with an SDHB mutation. J Clin Oncol 2011;29:e200e202.

62. Weber A, Hoffmann MM, Neumann HP, Erlic Z. Somatic mutation analysis of the SDHB, SDHC, SDHD, and RET genes in the clinical assessment of sporadic and hereditary pheochromocytoma. Horm Cancer 2012;3:187-192.

63. Habano W, Sugai T, Nakamura S, et al. Reduced expression and loss of heterozygosity of the SDHD gene in colorectal and gastric cancer. Oncol Rep 2003;10:1375-1380.

64. Carney JA. Carney triad. Front Horm Res 2013;41:92-110.

65. Haller F, Moskalev EA, Faucz FR, et al. Aberrant DNA hypermethylation of SDHC: a novel mechanism of tumor development in Carney triad. Endocr Relat Cancer 2014;21:567-577.

66. Piruat JI, Pintado CO, Ortega-Sáenz P, Roche M, López-Barneo J. The mitochondrial SDHD gene is required for early embryogenesis, and its partial deficiency results in persistent carotid body glomus cell activation with full responsiveness to hypoxia. Mol Cell Biol 2004;24:10933-10940.

67. Bayley JP, van Minderhout I, Hogendoorn PC, et al. Sdhd and SDHD/H19 knockout mice do not develop paraganglioma or pheochromocytoma. PLoS One 2009;4:e7987.

68. Millán-Uclés A, Díaz-Castro B, García-Flores P, et al. A conditional mouse mutant in the tumor suppressor SdhD gene unveils a link between p21(WAF1/Cip1) induction and mitochondrial dysfunction. PLoS One 2014;9:e85528.

69. Hoekstra AS, Bayley JP. The role of complex II in disease. Biochim Biophys Acta 2013;1827:543-551.

70. Goldstein AM. Germline BAP1 mutations and tumor susceptibility. Nat Genet 2011;43:925-926.
71. Alimonti A, Carracedo A, Clohessy JG, et al. Subtle variations in Pten dose determine cancer susceptibility. Nat Genet 2010;42:454-458.

72. Gaujoux S, Tissier F, Ragazzon B, et al. Pancreatic ductal and acinar cell neoplasms in Carney complex: a possible new association. J Clin Endocrinol Metab 2011;96:E1888-E1895.

73. Oishi $Y$, Nagai $S$, Yoshida M, et al. Mutation analysis of the SDHB and SDHD genes in pheochromocytomas and paragangliomas: identification of a novel nonsense mutation (Q168X) in the SDHB gene. Endocr J 2010;57: 745-750.

74. Cadiñanos J, Llorente JL, de la Rosa J, et al. Novel germline SDHD deletion associated with an unusual sympathetic head and neck paraganglioma. Head Neck 2011;33:1233-1240.

75. Hes FJ, Weiss MM, Woortman SA, et al. Low penetrance of a SDHB mutation in a large Dutch paraganglioma family. BMC Med Genet 2010;11:92.

76. Solis DC, Burnichon $\mathrm{N}$, Timmers $\mathrm{HJ}$, et al. Penetrance and clinical consequences of a gross SDHB deletion in a large family. Clin Genet 2009;75:354-363.

77. Dahia PL. Pheochromocytoma and paraganglioma pathogenesis: learning from genetic heterogeneity. Nat Rev Cancer 2014;14:108-119.

78. van Nederveen FH, Korpershoek E, Lenders JW, de Krijger RR, Dinjens WN. Somatic SDHB mutation in an extraadrenal pheochromocytoma. N Engl I Med 2007;357:306-308.

79. Gimm O, Armanios M, Dziema H, Neumann HP, Eng C. Somatic and occult germ-line mutations in SDHD, a mitochondrial complex II gene, in nonfamilial pheochromocytoma. Cancer Res 2000;60:6822-6825.

80. Killian JK, Kim SY, Miettinen M, et al. Succinate dehydrogenase mutation underlies global epigenomic divergence in gastrointestinal stromal tumor. Cancer Discov 2013;3:648-657.

81. Neumann HP, Pawlu C, Peczkowska M, et al.; European-American Paraganglioma Study Group. Distinct clinical features of paraganglioma syndromes associated with SDHB and SDHD gene mutations. JAMA 2004;292:943-951.

82. Benn DE, Gimenez-Roqueplo AP, Reilly JR, et al. Clinical presentation and penetrance of pheochromocytoma/paraganglioma syndromes. J Clin Endocrinol Metab 2006;91:827-836. 